The content of the book may be summarised as follows: the text addresses all aspects of fatigue from a materials science point of view. The author traces the life cycle of cracks from gestation, initiation, propagation to ultimate component failure. The text is highly mathematical and described by the author as a study in analytical fracture mechanics, the core content of which is derived from the synthesis of microand macro-mechanics of fatigue - that is at the level of grains, fibres, microcracks and pores. As such, consideration of crystal lattice defects and other metallurgical issues is excluded, prompting the reviewer to recommend G. N. Haidemenopoulos's Physical Metallurgy: Principles and Design (CRC Press, 2018) as a companion volume, albeit much more expensive. But this in no way detracts from the value of this highly informative/thought-provoking book.

Peter C. Gasson, CEng MIMechE, FRAeS

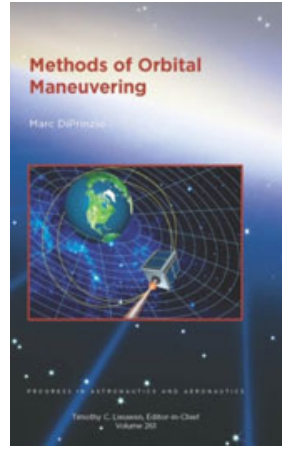

Methods of Orbital Manoeuvring, Progress in Aeronautics and Astronautics series volume 261

\section{Di Prinzio}

American Institute of Aeronautics and Astronautics, Reston, VA, USA. xiv; 1238 pp. 2019. Illustrated. Distributed by Transatlantic Publishers Group, 97 Greenham Road, London N10 1LN, UK. $£ 131$ (20\% discount available to RAeS members on request; mark.chaloner@ tpgltd.co.uk). ISBN 978-1-62410-582-1.

t 1,238 pages, Methods of Orbital $\triangle$ Manoeuvring is a magnum opus. 1 One reason for the book's size is that it is packed with helpful example calculations, in which the mathematics is well explained. If you are responsible for 'manoeuvring' (or even 'maneuvring') a satellite that uses chemical propellants and you need to manage its on-board propellant effectively, this is the reference work for you. 
You will need some background knowledge, however. The book opens with an introduction that points the reader towards other reference works that might aid comprehension. Some of the assumed knowledge is reasonable - the Keplerian orbital parameters, for example, are very well known - but other parameters (some of which are used repeatedly in the early chapters) could have been explained in the text. 'Argument of latitude' is arguably a concept for orbit aficionados, and 'semi parameter' is a term that is far more familiar as 'semi-latus rectum' in the orbital literature. A preliminary search for this neologism on the internet failed to generate a reference, at which point one does start to wonder whether, somewhere in those 1,238 pages, there might have been room for a small explanatory diagram.

The early chapters of the book deal with singe-impulse transfers - transfers between two points (any fans of Lambert's problem will not be disappointed), techniques for identifying two-impulse strategies for transfers between orbits and then strategies for optimising these transfers. Towards the end of this fifth chapter there is a discussion of the trade-off between the computer runtimes associated with different optimisation algorithms and the accuracy of the solutions found. While potentially still relevant, this debate is likely to become much less of an issue as computing power increases.

Chapter 6 on 'Alternate Lambert Techniques' is where the author starts to introduce real-world constraints into the largely theoretical discussion that has gone before; Sun angle limitations and pitch and yaw angle considerations make their first appearance in the text at this point. A 'rite of passage' for all orbital dynamics geeks is to discover John Draim's paper which describes a global coverage constellation using just four satellites, and hence it is wholly appropriate that one of the examples in this chapter involves a transfer to a Draim orbit. Well played, sir! Chapter 6 is also home to one of the slight oddities in the volume. As you might expect in a scientific textbook, it is largely in the third person (indeed, in chapter 3 the author cites one of his own research papers as 'DiPrinzio 2012'); however, on page 282 there is an incongruous paragraph written in the first person which escaped the 'blue pencil'.

The next chapter introduces more constraints on manoeuvres, including limits on the admissible values of the various orbital parameters. It is in this chapter too that the issue of manoeuvre duration makes its first appearance - a recognition of the fact that changes to a satellite's orbital velocity do not happen truly impulsively but take a finite time to achieve. In chapter 8 , a series of useful two-impulse case studies are discussed, including manoeuvres to reach a 24-h-period, highly inclined, highly elliptical trajectory known as a 'Tundra' orbit. Two references are provided at this point in the text (the first citing a second earlier work from 2001); this reads like a search for the genesis of this orbit concept. If $\mathrm{Mr}$ DiPrinzio ever encounters this review of his masterwork, he may find 'The Tundra Orbit. A Communications Satellite Constellation Optimised for Continuous Coverage of the Whole of Canada.' DCDS Space Satellite Office, NDHQ Ottawa, 16 March 1981' of interest - the idea is at least two decades older. Another slight oddity is that, despite naming the Tundra orbit, its semi synchronous cousin, the Molniya orbit, is referred to as '12-Hour HEO' throughout. There are very few inaccuracies in the 
text, but the suggestion on page 426 that 'free-drift' Geosynchronous Equatorial Orbit (GEO) missions (where the orbital inclination is allowed to evolve over time) have an initial right ascension of the ascending node (RAAN) of $270^{\circ}$ is somewhat misleading. Due to the motion of the orbit pole over time, the initial RAAN value is typically closer to $285^{\circ}$, depending on the planned lifetime of the satellite and its initial inclination. This is, however, an area where the book is likely to be overtaken by technology. The advent of high-specific-impulse electric propulsion means that north-south station keeping on GEO satellites is now much easier to achieve, with the result that very few satellite operators will need to plan 'free-drift' missions in the future.

Chapter 9 is comparatively short and deals with the optimisation of orbits. It introduces the concept of acceptable tolerances on the orbit parameters and provides more useful examples involving regularly used orbits (in this case, the parameters for semisynchronous GPS navigation). In chapter 10 , the concept of three-impulse transfers is introduced. The aforementioned orbital dynamics geeks who have been holding their breath, eagerly waiting for 'bi-elliptic transfers', get to breathe a sigh of relief at this point. A range of constraints on such transfers are discussed (including inclination and RAAN), but curiously 'transfer time' is not one of them but perhaps should have been. As a general principle, three burns typically take longer than two burns to achieve the final orbit, but in some cases the total propellant expended can be less, especially when large inclination changes are required. A clear opportunity to utilise bi-elliptic transfers was when Russia was first seeking to insert satellites into GEO using the Proton rocket from Baikonur. Due to the latitude of the launch site, and a desire to avoid overflying China, Russian GEO transfer orbits had an inclination of $52^{\circ}$, and a bi-elliptical strategy could have saved them much propellant when trying to get their satellites into final orbits with inclinations close to $0^{\circ}$. The fact that the Russians did not choose to adopt this approach is due to the fact that their satellites were running on batteries prior to the deployment of the solar panels, and the bi-elliptic approach would have simply taken too long. Time clearly is an important parameter here.

The subjects addressed in chapter 11 include finite burn times, multiple manoeuvres and the need to address orbital perturbations (due to the Earth's asphericity, for example), to enable high-fidelity orbital dynamics. Chapter 12 continues the theme of high-fidelity algorithms and introduces the concept of equinoctial orbital elements, modifications to the traditional Keplerian formulation to address the possible singularities that can occur when the orbital inclination or eccentricity is close to zero.

Chapter 13 is one of the most useful in the book, with more than 100 pages discussing common mission constraints. Principal among these is the subject of eclipses, which obviously affect a satellite's ability to generate power, but also influence its thermal control and, in some cases, its ability to determine its orientation if sun sensors are the basis of its attitude control system. Avoiding long eclipses is thus a key objective of a mission planner.

We return in chapter 14 to the issue of optimisation of high-fidelity orbital transfers, and the fact that, by planning a series of manoeuvres, an operator can compensate for errors in the applied velocity changes. This recognition of real-world issues (in 
which rockets do not always behave quite as expected) is one of the book's strengths. This chapter also includes text on a further possible constraint, radio-frequency interference (which might have been more at home a chapter earlier). Again, though, it is the sort of practical issue that mission operators need to take into account.

The final chapter is a very comprehensive case study example of a fictional mission design called BTSat (since it is designed to monitor the Bermuda Triangle from GEO orbit!). The reader can learn a lot from this chapter, including how to manage errors of various types, and how to plan contingencies to accommodate them. You might think that sending a GEO satellite with a constrained propellant budget around the Moon to get it on station sounds somewhat implausible, but this triumph of orbital dynamics with the HGS-1 satellite gets an honorary mention here too. There is even room for a useful section on the perennially difficult problem of propellant estimation on satellites, which becomes especially relevant as the need to perform efficient GEO graveyard burns is recognised.

A couple of final notes: There are some topics that this book does not cover; for example, ion propulsion (which involves very high-specific-impulse, low-thrust trajectories) is not addressed. All the examples given are for satellites equipped with chemical propulsion. The march of technology towards 'all-electric' satellite designs may render some of the examples in this textbook redundant (although the principles will remain true). Also, the satellites discussed are treated as independent entities, whereas the rise of mega-constellations involves many satellites working together and needing to maintain relative station in an efficient manner. This is another rich seam that has not been mined here. A final point for the publishers AIAA is that at least four pages in this review copy attempted to part company with the book on a first reading. If this book is used frequently as a valuable reference source (which it deserves to be), it has the potential to disintegrate. As noted above, the book has 1,238 pages; it definitely merits more glue!

Dr Stuart Eves 